\title{
Renal leiomyoma: a case report and literature review
}

\author{
Sajal Gerdharee ${ }^{1}$, Abrie van Wyk ${ }^{2}$, Heidi van Deventer ${ }^{1 *}$ (D) and Andre van der Merwe ${ }^{1}$
}

\begin{abstract}
Background: Renal leiomyomas are exceptionally rare, benign, mesenchymal tumours originating from smooth muscle in the kidney. Historically, because of their small size, most renal leiomyoma cases were discovered incidentally based on autopsy findings. However, since the advent and improved access to imaging modalities such as ultrasound and computed tomography (CT), renal leiomyomas are being discovered more frequently. Although usually incidental discoveries, clinical presenting signs and symptoms comprise abdominal or flank pain, a palpable flank mass, and haematuria in $20 \%$ of those with symptoms.

Case presentation: We study the case of an incidentally found, asymptomatic, left kidney mass that presented in a 60 -year-old female. Initial suspicions on CT imaging of either renal cell carcinoma or oncocytoma resulted in a radical nephrectomy of the left kidney. Postoperative pathological examination of the mass revealed a renal leiomyoma; a rare, benign tumour that is mostly indistinguishable from malignant tumours on imaging.

Conclusions: With the current availability of ultrasonography and CT, they are often discovered incidentally, and the radiological differential diagnoses are often inadequate or challenging in such cases. The gold standard management of these suspicious cancer cases is still a radical nephrectomy with postoperative pathological and immunohistochemical analysis. Due to its benign nature, patients enjoy excellent prognoses without recurrence. We discuss and briefly review the relevant literature of the clinical, imaging and pathological features of renal leiomyomas and those of the differential diagnoses.
\end{abstract}

Keywords: Renal leiomyoma, Case report, Renal leiomyoma diagnosis, Literature review, Renal leiomyoma imaging

\section{Background}

Renal leiomyomas are exceptionally rare, benign, mesenchymal tumours originating from smooth muscle in the kidney. Renal smooth muscle can be found within the renal capsule, calyces, pelvis, and blood vessels; hence, any of these areas can be a site of origin for leiomyomas.

Steiner and colleagues found that of symptomatic leiomyomas, $53 \%$ of tumours were localised subcapsular, $37 \%$ capsular, and $10 \%$ within the renal pelvis [1].

Historically, because of their small size, most renal leiomyoma cases were discovered incidentally based

\footnotetext{
*Correspondence: heidivd@sun.ac.za

${ }^{1}$ Division of Urology, Faculty of Medicine and Health Sciences, Tygerberg Hospital, Stellenbosch University, P.O. Box 19063, Tygerberg 7505, South Africa

Full list of author information is available at the end of the article
}

on autopsy findings [2]. However, since the advent and improved access to imaging modalities such as ultrasound and CT, renal leiomyomas are being discovered more frequently.

Although usually incidental discoveries, clinical presenting signs and symptoms comprise abdominal or flank pain, a palpable flank mass, and haematuria in $20 \%$ of those with symptoms. Cases suggest a higher prevalence in adult females, with an average age of 42 at presentation [1,3]. Leiomyomas of the kidney are even rarer in the paediatric population, and there have been very few reported cases of these tumours in the literature. Some cases of interest included a six-yearold boy with an initially suspected Wilms tumour; a leiomyoma occurring in a transplanted kidney in association with the Epstein-Barr virus (EBV), five years 
post renal transplant; and a vascular renal leiomyoma in an 11-year-old with bilateral RCC [4].

Over ten years, the Brady Urological Institute reviewed several consecutive nephrectomies as a result of renal tumours. Of a total of 1030 nephrectomies, renal leiomyomas comprised $0.3 \%$ of the total, and $1.5 \%$ of all benign tumours removed [5].

In terms of etiological factors leading to the development of a renal leiomyoma, there are various theories and suggested causative factors. Krishnan and colleagues showed a possible causal relationship between Epstein-Barr virus and renal leiomyoma in immunocompromised patients, while Tsujimura and colleagues suggested a possible link with tuberous sclerosis; a condition more commonly associated with renal angiomyolipoma (AML) $[6,7]$.

When considering the relevant differential diagnosis concerning clinical presentation and imaging studies, three renal tumours that are more commonly found are $\mathrm{AML}$, oncocytoma, and the diagnostic importance that lies in distinguishing them from the malignant RCC [8]. Renal cell carcinomas make up the majority of contrast-enhancing small renal masses, the most prevalent histological type being the clear cell RCC. Frank and colleagues demonstrated a clear cell RCC incidence of $55 \%$ after 2770 surgeries (radical nephrectomies or nephron-sparing) for solid, unilateral, nonmetastatic tumours [9].

AMLs are benign tumours in the perivascular epithelioid cell tumour family, comprising variable mixtures of thick-walled blood vessels, mature fat, and smooth muscle. It may sometimes be represented by dominance of the smooth muscle component, hence its likeness to a leiomyoma. Furthermore, they are characterised by smooth muscle and melanocytic marker (HMB-45) co-expression [10]. In contrast to this, renal leiomyoma tumour cells typically test negative for HMB-45, but express smooth muscle actin and desmin. Angiomyolipoma is also relatively well-distinguished radiologically as an enhancing mass with macroscopic fat, devoid of calcification. Small, fat-poor tumours can be more challenging to distinguish from RCC [11].

Oncocytoma often displays the appearance of a wellcircumscribed, homogeneous, and solid lesion, and may often demonstrate central scarring-features that are not mutually exclusive with the presentation of RCC [12]. Postoperatively the pathologist also needs to differentiate leiomyoma from leiomyosarcoma [10]. Histological features associated with leiomyosarcoma are tumour necrosis, nuclear pleomorphism, and increased mitotic activity [13].

\section{Case presentation}

\subsection{Clinical history}

The patient under study is a 60 -year-old female, previously diagnosed with hypertension, diabetes mellitus (type II) and congestive cardiac failure. After a routine chest radiograph displayed suspicious nodularity in both lung apices, a CT was performed, and the nodules were queried to be post-infective changes or suspicious of metastases. An abdominal CT was then performed in search of a primary neoplastic lesion and a mixed, solidcystic mass in the lower pole of the left kidney was discovered. The mass was asymptomatic, and she had no associated pain, discomfort or haematuria.

\subsection{Investigations}

The abdominal CT showed a large solid/cystic tumour in the lower pole of the left kidney. The dimensions of the renal mass was $9 \mathrm{~cm} \times 8 \mathrm{~cm}$, demonstrating peripheral soft tissue enhancement with a central, cystic component measuring $12 \mathrm{HU}$ (Hounsfield Units). It was highly suspicious of an RCC with central necrosis. Two months later, a repeat contrasted CT was done to evaluate for the progression of the suspicious lung lesions, which may have indicated metastases, and for the appearance of new intra-abdominal metastases. CT findings were unchanged from before, as seen in Figs. 1 and 2.

There was displacement of the lower pole of the kidney with marked compression of the proximal ureter, which was splayed around the mass-resulting in mild hydronephrosis. The mass demonstrated fat stranding and bordered the retroperitoneal fascia but did not bridge or enter the peritoneal cavity. Similarly, renal vessels were splayed but not infiltrated, with no signs of neovascularisation. There was no clear claw sign demonstrated to suggest renal origin, and no associated lymphadenopathy or distant metastases. These features were not in keeping with an aggressive RCC, and a possible diagnosis of a renal oncocytoma was considered. However, it is noted that the absence of a renal claw sign and clear origin from the renal cortex remains atypical for either of the diagnoses mentioned.

The patient was prepared for surgery and underwent a radical nephrectomy. The postoperative course was uneventful, and the patient was discharged.

Histological sections of the tumour displayed a wellcircumscribed, unencapsulated mass comprising spindled cells with a fascicular growth pattern (Fig. 3). There was central cystic degeneration, associated with degenerative atypia but without any mitotic figures noted. There was no evidence of vascular invasion or necrosis. Immunohistochemically, the lesion was strongly positive for desmin and smooth muscle actin and negative for 

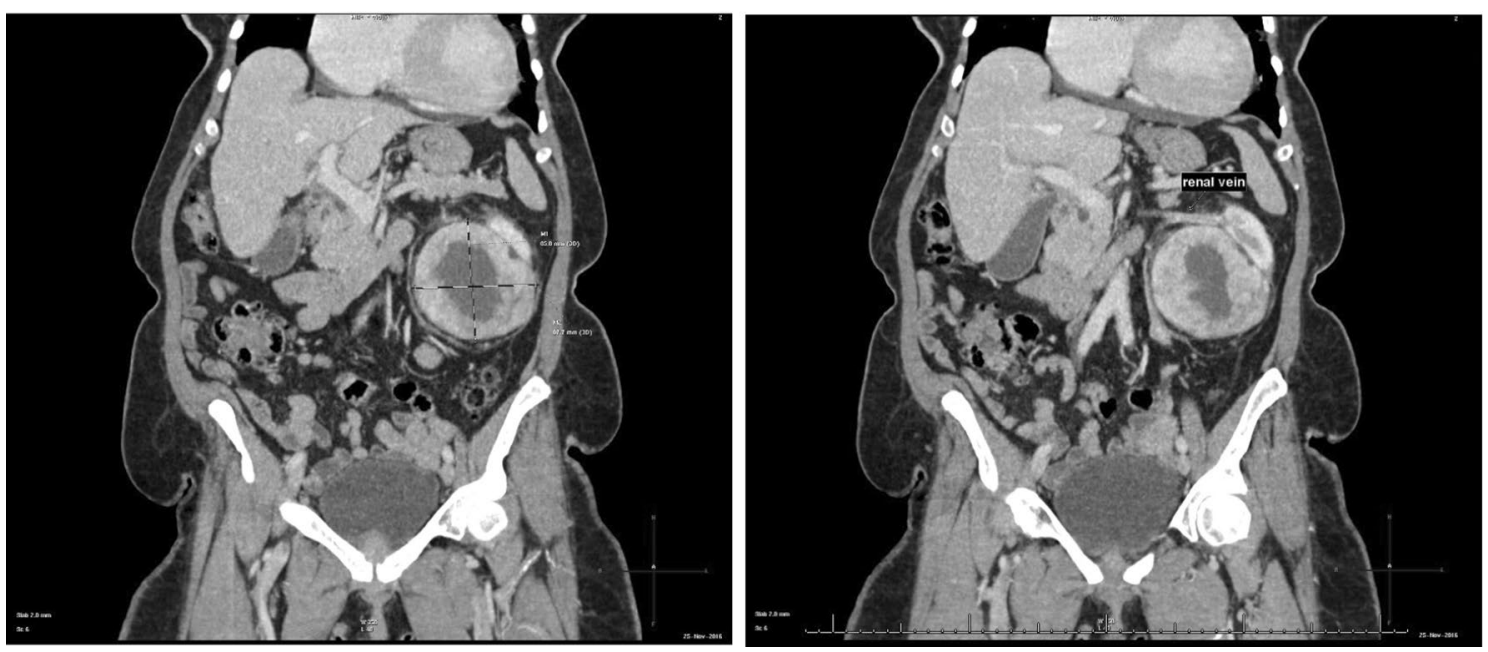

Fig. 1 Abdominal CT demonstrating renal mass
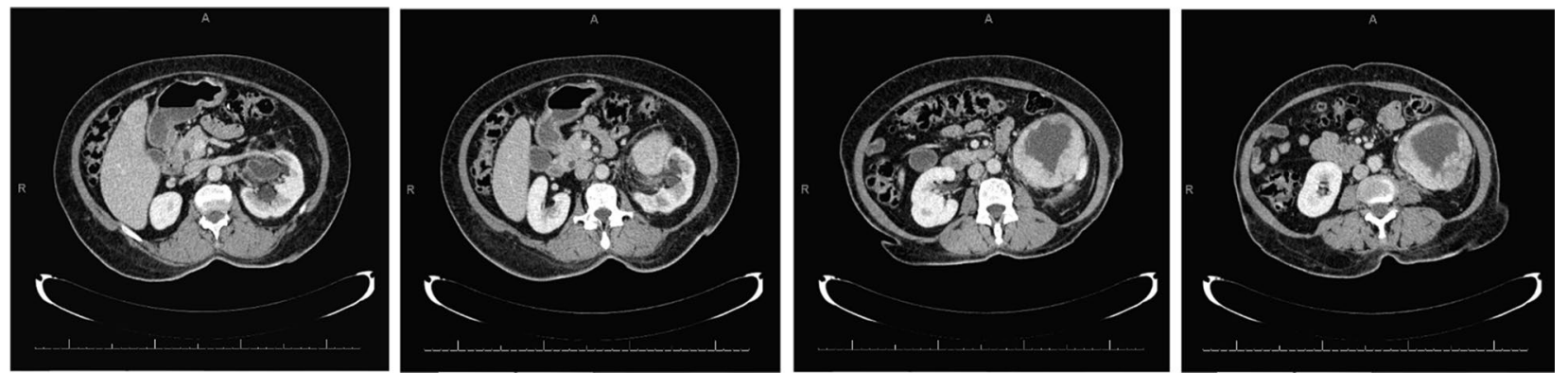

Fig. 2 Abdominal CT demonstrating renal mass

HMB-45 and melan A. Chromogenic in situ hybridisation for Epstein-Barr encoded RNA was negative, excluding EBV associated smooth muscle neoplasm, which may have similar morphology. These results, in keeping with clinically absent features of tuberous sclerosis, favoured a diagnosis of a renal leiomyoma. There was unfortunately no mention made by the histological report of whether the tumour originated from capsular or subcapsular renal tissue.

\section{Discussion}

The literature reviewed comprised entirely of case studies that included a diagnosis of renal leiomyoma, within no specific time period. Provided that the diagnosis of a renal leiomyoma was made, there were no other exclusion criteria other than the patient's age being older than 16 years-of which no such cases were eliminated thereby. Cases were reviewed and added on a case-tocase basis as they were found. Search engines utilised in
English, comprised primarily of PubMed, and the university library e-databases.

We reviewed 20 cases of renal leiomyomas in adults (19 from the literature and one of our own), summarised in Table 1 . The mean age was 49 , with a $70 \%$ predominance of female patients. Sixty-eight per cent of these cases were symptomatic before the discovery of their renal leiomyomas. Of those cases reporting signs, 53\% had abdominal pain, $32 \%$ had a flank mass, while only $26 \%$ were haematuric. Only one of nineteen presented with all three clinical features simultaneously, while $32 \%$ had at least two of the features present. In cases where tumour origin was mentioned, there was a $50 \%$ predominance of capsular leiomyomas, and only two cases were located in the subcapsular area. Of the cases that reported immunohistochemical testing, $100 \%$ of tumours tested negative for HMB-45. All the tumours that were tested for smooth muscle actin and/or desmin were positive for these markers. Tumour sizes ranged from 1.3 to $32 \mathrm{~cm}$, with an average diameter of $9.6 \mathrm{~cm}$. 

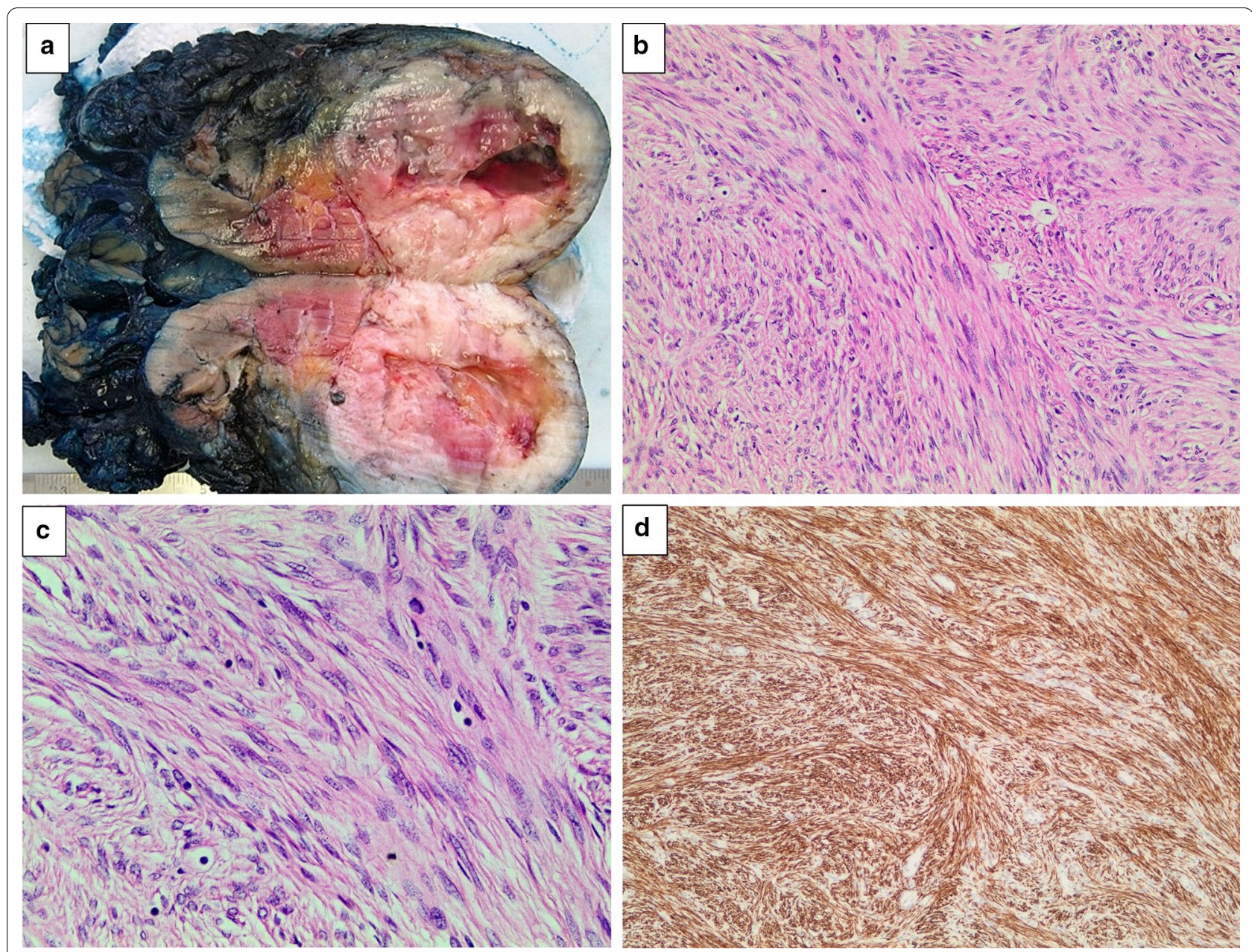

Fig. 3 a Left kidney bisected. A round, well-circumscribed tumour with central cystic degeneration is present in the lower pole of the kidney. $\mathbf{b}$ The tumour is composed of spindle-shaped cells with eosinophilic cytoplasm growing in intersecting fascicles. Haematoxylin and eosin, original magnification $\times 200$. c The nuclei of the tumour cells often have rounded ends ("cigar-shaped" nuclei). Scattered lymphocytes are noted in the background. Significant pleomorphism, tumour necrosis and increased mitotic activity are absent. Haematoxylin and eosin, original magnification $\times 400$. d An immunohistochemical stain for desmin shows strong and diffuse cytoplasmic staining. Original magnification $\times 200$

Some of these results differ slightly from the literature represented earlier concerning presentation, which is likely due to the sample size and lack of reporting on the various clinical and pathological characteristics in many of these cases.

\subsection{Pre-operative diagnostics}

On ultra-sonographic imaging, leiomyomas typically appear hypoechoic with either a solid or a cystic appearance [10]. Some helpful CT features were pointed out by Derchi and colleagues to help with the differential diagnosis. Firstly, the density before contrast of all leiomyomas displayed a likeness to that of muscle and was hyper-dense. After the addition of contrast, the masses had lower enhancement in comparison with the renal parenchyma. Secondly, the lesions display no infiltration of neighbouring tissue and are usually well-circumscribed and located peripherally. These features see the addition of a renal leiomyoma to the differential; however, malignant disease still cannot be excluded. The presence of infiltration virtually excludes a benign renal leiomyoma $[1,14]$.

The size and enhancement of incidentally discovered, small, asymptomatic renal masses may be of value in determining the likely nature of such lesions.

A study of 2770 renal tumours found that almost half the number of non-metastatic tumours smaller than $1 \mathrm{~cm}$ were benign, while tumours of $7 \mathrm{~cm}$ or larger comprised only $6.3 \%$ of benign tumours [9]. O'Connor and colleagues discovered incidental renal lesions greater 
Table 1 Clinicopathological variations of renal leiomyomas diagnosed in adults

\begin{tabular}{|c|c|c|c|c|c|c|c|c|c|c|c|}
\hline Case reference & Age & Sex & Symptomatic & $\begin{array}{l}\text { Heamaturia } \\
\text { (microscopic) }\end{array}$ & Flank mass & $\begin{array}{l}\text { Abdominal } \\
\text { pain }\end{array}$ & Origin & HMB-45 & SMA & Desmin & Size $(\mathrm{cm})$ \\
\hline$\# 1[1]$ & 57 & $\mathrm{~F}$ & No & No & No & No & Subcapsular & - & - & - & $11 \times 6$ \\
\hline \#2 [1] & 46 & $M$ & Yes & No & Yes & No & Cortex & - & - & - & $22 \times 17 \times 10$ \\
\hline \#3 [1] & 67 & $\mathrm{~F}$ & Yes & No & No & Yes & Capsular & - & - & - & $7 \times 6 \times 6$ \\
\hline \#4 [1] & 59 & $\mathrm{~F}$ & No & Yes & No & No & Subcapsular & - & - & - & $1.3 \times 1.2 \times 0.7$ \\
\hline \#5 [3] & 53 & M & Yes & No & Yes & Yes & - & - & - & - & $30 \times 29 \times 27$ \\
\hline \#6 [5] & 62 & $\mathrm{~F}$ & No & No & No & No & - & Neg & - & Pos & 1.8 \\
\hline \#7 [5] & 59 & $\mathrm{~F}$ & Yes & No & No & Yes & - & - & - & - & 2 \\
\hline \#8 [5] & 42 & $\mathrm{~F}$ & No & No & No & No & - & - & - & Pos & 3.6 \\
\hline \#9 [10] & 31 & M & Yes & Yes & No & Yes & - & Neg & Pos & - & $8.6 \times 11$ \\
\hline \#10 [17] & 40 & $\mathrm{~F}$ & Yes & Yes & No & Yes & Capsular & Neg & Pos & Pos & $9.5 \times 7.5$ \\
\hline \#11 [17] & 27 & M & Yes & Yes & Yes & Yes & - & - & - & - & $3 \times 3$ \\
\hline \#12 [17] & 52 & $F$ & Yes & Yes & No & Yes & Capsular & - & Pos & - & $8 \times 7$ \\
\hline \#13 [18] & 39 & M & Yes & No & Yes & No & - & Neg & Pos & Pos & $11 \times 9.5 \times 9.5$ \\
\hline \#14 [19] & 56 & $F$ & Yes & No & No & Yes & - & - & Pos & Pos & $8 \times 5.5$ \\
\hline \#15 [20] & 41 & $\mathrm{~F}$ & Yes & No & No & Yes & Renal vein wall & Neg & Pos & - & $4 \times 3.5$ \\
\hline \#16 [21] & 45 & $\mathrm{~F}$ & Yes & No & Yes & Yes & Capsular & - & Pos & - & $32 \times 25 \times 12$ \\
\hline \#17 [22] & 40 & M & Yes & No & Yes & No & - & - & - & - & $10 \times 9 \times 7.5$ \\
\hline \#18 [23] & 43 & $\mathrm{~F}$ & No & No & No & No & Capsular & Neg & Pos & Pos & $3.8 \times 3.2 \times 2.8$ \\
\hline$\# 19$ [24] & 56 & $\mathrm{~F}$ & - & - & - & - & Renal pelvis & Neg & - & Pos & 3.2 \\
\hline \#20 & 60 & $\mathrm{~F}$ & No & No & No & No & - & Neg & Pos & Pos & $8 \times 9$ \\
\hline
\end{tabular}

SMA, smooth muscle actin; HMB-45, human melanoma black; F, female; M, male; -, unknown/unreported, Neg, negative, Pos, positive

than $1 \mathrm{~cm}$ in 433 of 3001 (14\%) in asymptomatic adults after an abdominopelvic CT for screening colonography. Of these, $87 \%$ were radiologically characterised as benign and a further $13 \%$ of "indeterminate significance" [15]. Radiologically, the enhancement of a renal lesion is considered a valuable characteristic in determining the nature of the lesion. While there is some consensus that enhancement of $20 \mathrm{HU}$ or more is significant in identifying malignant lesions, some authors suggest that lesions displaying $10 \mathrm{HU}$ or more should still be considered significantly suspicious of malignancy. Therefore, lesions displaying attenuation from $10 \mathrm{HU}$ to $19 \mathrm{HU}$ are considered "indeterminate".

This determination has some implications when considering the possibility of pre-operative diagnosis in the form of CT-guided core biopsies. Although it remains controversial, Eshed and colleagues believe that due to the low complication rate and value in implementing disease-tailored management, indication for core biopsies should include radiographically indeterminate lesions and small renal masses. In agreement, Romero and colleagues suggest that there is a place for biopsy and conservative management in patients with radiographic and clinical evidence of a diagnosis other than
RCC. These lesions with benign biopsy results can then undergo resection, re-evaluation or close clinical or imaging surveillance $[5,16]$. Where availability and resources allow for such, the use of Magnetic Resonance Imaging (MRI) in the diagnosis of renal masses is another area of diagnostics which may benefit preoperative diagnostics. However, in a resource-poor setting, this modality is a less feasible option than CT due to limited availability in the public sector, and cost. It is thus not further discussed in this paper.

Currently, management of larger lesions still holds surgical resection as its gold standard, with radical nephrectomy being the usual approach to suspicious lesions. Lesions equal to, or smaller than, $4 \mathrm{~cm}$ allow consideration for nephron-sparing resections, and smaller renal masses can be biopsied-although still considered a somewhat controversial practice [1]. In the ideal setting, nephron-sparing resection could also be a consideration, given the availability of extemporaneous examination during the surgical procedure. Likewise, the same remains true with regard to performing an initial renal biopsy for immunohistological diagnosis. The indications for either of which would also need to be correlated with pre-operative imaging findings. 


\section{Conclusion}

Accurate detection of renal leiomyomas may be challenging in the pre-operative setting, given the variety of findings on imaging. There are very few characteristics that may help rule in or rule out the leiomyoma amongst its differential, especially when suspicious or malignant lesions have to be considered. Pre-operative, imageguided core biopsy would undoubtedly aid in distinguishing the leiomyoma. However, its use is dependent on multiple factors ranging from clinical presentation to size and contrast enhancement of the lesion, and availability of resources. In addition to being viewed as a controversial practice, the place for biopsy in the resource-poor setting is yet to be determined. Until more rigid indications are established surrounding conservative treatment, definitive management will invariably be surgical, as these patients enjoy an excellent prognosis without recurrence.

\section{Abbreviations}

AML: Angiomyolipoma; CT: Computed tomography; EBV: Epstein-Barr virus; HU: Hounsfield units; RCC: Renal cell carcinoma.

\section{Acknowledgements}

None.

\section{Authors' contributions}

SG did the write up of the case report and literature review as part of his undergraduate studies. HVD and AVDM contributed equally to the writing of the manuscript. AVW reviewed the pathology section of the manuscript. All authors read and approved the final manuscript.

\section{Funding}

No funding was received for this study.

\section{Availability of data and materials}

Data sharing does not apply to this article as no datasets were generated or analysed during the current study.

\section{Ethics approval and consent to participate}

Ethics approval was obtained from the Health Research Ethics Committee of Stellenbosch University with the reference number of UC19/10/048. Consent to participate is not applicable.

\section{Consent for publication}

Written informed consent for publication of the patient's clinical details and clinical images was obtained from the patient.

\section{Competing interests}

The authors declare that they have no competing interests.

\section{Author details}

${ }^{1}$ Division of Urology, Faculty of Medicine and Health Sciences, Tygerberg Hospital, Stellenbosch University, P.O. Box 19063, Tygerberg 7505, South Africa. ${ }^{2}$ Division of Anatomical Pathology, NHLS Tygerberg and University of Stellenbosch, Cape Town, South Africa.

Received: 27 November 2019 Accepted: 12 February 2021

Published online: 25 February 2021

\section{References}

1. Steiner M, Quinlan D, Goldman S, Millmond S, Hallowell M, Stutzman R et al (1990) Leiomyoma of the kidney: presentation of 4 new cases and the role of computerized tomography. J Urol 143(5):994-998

2. Belis J, Post G, Rochman S, Franklin MD (1979) Genitourinary leiomyomas. Urology 13(4):424-429

3. Andreoiu M, Drachenberg D, Macmahon R (2009) Giant renal leiomyoma: a case report and brief review of the literature. Can UrolAssoc J 3(5):58-60

4. Nakib G, Mahgoub N, Calcaterra V, Pelizzo G (2017) Renal leiomyoma in pediatric age: a rare case report with review of the literature. J PediatrSurg Case Rep 27:43-46

5. Romero F, Kohanim S, Lima G, Permpongkosol S, Fine S, Kavoussi L (2005) Leiomyomas of the kidney: emphasis on conservative diagnosis and treatment. Urology 66(6):1319.e1-3

6. Krishnan R, Freeman J, Creager A (1999) Epstein-Barr virus induced renal leiomyoma. J Urol 161(1):212

7. Tsujimura A, Miki T, Sugao H, Takaha M, Takeda M, Kurata A (1996) Renal leiomyoma associated with tuberous sclerosis. Urollnt 57(3):192-193

8. Nagar A, Raut A, Narlawar R, Bhatgadde V, Rege S, Thapar V (2004) Giant renal capsular leiomyoma: study of two cases. Br J Radiol 77(923):957-958

9. Frank I, Blute M, Cheville J, Lohse C, Weaver A, Zincke H (2003) Solid renal tumors: an analysis of pathological features related to tumor size. J Urol 170(6 Pt 1):2217-2220

10. Brunocilla E, Pultrone C, Schiavina R, Vagnoni V, Caprara G, Martorana G (2012) Renal leiomyoma: case report and literature review. Can UrolAssoc J 6(2):E87-90

11. Kim J, Park S, Shon J, Cho K (2004) Angiomyolipoma with minimal fat: differentiation from renal cell carcinoma at biphasic helical CT. Radiology 230(3):677-684

12. Perez-Ordonez B, Hamed G, Campbell S, Erlandson RA, Russo P, Gaudin PB et al (1997) Renal oncocytoma: a clinicopathologic study of 70 cases. Am J SurgPathol 21(8):871-883

13. Hogan A, Smyth G, D'Arcy C, O'Brien A, Quinlan D (2008) Renal capsular leiomyoma. Urology 71(6):1226.e1-3

14. Derchi L, Grenier N, Heinz-Peer G, Dogra V, Franco F, Rollandi G et al (2008) Imaging of renal leiomyomas. ActaRadiol 49(7):833-838

15. O'Connor S, Pickhardt P, Kim D, Oliva M, Silverman S (2011) Incidental Finding of renal masses at unenhanced CT: prevalence and analysis of features for guiding management. Am J Roentgenol 197(1):139-145

16. Eshed I, Elias S, Sidi A (2004) Diagnostic value of CT-guided biopsy of indeterminate renal masses. ClinRadiol 59(3):262-267

17. Mitra B, Debnath S, Pal M, Paul B, Saha T, Maiti A (2012) Leiomyoma of kidney: an Indian experience with literature review. Int J Surg Case Rep 3(11):569-573

18. Larbcharoensub N, Limprasert V, Pangpunyakulchai D, Sanpaphant S, Wiratkapun C, Kijvikai K (2017) Renal leiomyoma: a case report and review of the literature. Urol Case Rep 7(13):3-5

19. Akbulut F, Şahan M, Üçpınar B, Savun M, Özgör F, Arslan B et al (2016) A rare case of renal leiomyoma treated with laparoscopic partial nephrectomy. Med Bull Haseki 54(1):41-43

20. Kocak C, Kabay S, Isler B (2015) Leiomyoma of the renal vein: a rare tumor presenting as a renal mass. Case Rep Urol 5(2015):1-3

21. Lal A, Galwa R, Chandrasekar P, Sachdeva M, Vashisht R, Khandelwal N (2009) A huge renal capsular leiomyoma mimicking retroperitoneal sarcoma. Saudi J Kidney Dis Transpl 20(6):1069-1071

22. Takezaki T, Nakama M, Ogawa A (1985) Renal leiomyoma with extensive cystic degeneration. Urology 25(4):401-403

23. Fu L, Humphrey P, Adeniran A (2015) Renal leiomyoma. J Urol 193(3):997-998

24. Patil P, McKenney J, Trpkov K, Hes O, Montironi R, Scarpelli M et al (2015) Renal leiomyoma: a contemporary multi-institution study of an infrequent and frequently misclassified neoplasm. Am J SurgPathol 39(3):349-356 